\title{
Detection of ultra-long-period oscillations in an EUV filament
}

\author{
C. Foullon, E. Verwichte, and V. M. Nakariakov
}

\author{
Department of Physics, University of Warwick, Coventry CV4 7AL, UK \\ e-mail: foullonc@astro.warwick.ac.uk
}

Received 23 June 2004 / Accepted 22 September 2004

\begin{abstract}
We report the first detection of long-period $(8-27 \mathrm{~h})$ oscillatory intensity variations in a coronal filament. The filament is observed continuously as it crosses the solar disk in a 12-min-cadence SoHO/EIT $195 \AA$ uninterrupted data set. Cyclic intensity variations are found to be correlated along the filament, while the most pronounced oscillations are detected at its southern end for nearly 6 days. The dominant period of these oscillations is $12.1 \mathrm{~h}$ and the amplitude of the intensity variations reaches approximately $10 \%$ of the background intensity. The ultra-long-period oscillations may be interpreted in terms of slow string MHD modes or may be connected with thermal over-stability associated with peculiarities of the cooling/heating function and with the effect of neutrals. These theoretical predictions however do not explain the spatial structure of the oscillations along the filament.
\end{abstract}

Key words. Sun: prominences - filaments - oscillations - UV radiation

\section{Introduction}

Since April 1997 the Extreme-ultraviolet Imaging Telescope (EIT) (Delaboudinière et al. 1995) on board the Solar and Heliospheric Observatory (SoHO) runs a "CME watch program", where images of the full Sun are taken, mostly at the wavelength of $195 \AA$, every 10 to $15 \mathrm{~min}$. Dynamical analysis of the $195 \AA$ images have so far focused on short-term events typically connected to eruptive phenomena, i.e. flares, filament eruptions and Coronal Mass Ejections (CMEs). These shortterm events are detected and studied in data series, which do not exceed an hour or a day (see e.g., Thompson et al. 1999). In this Letter, a dynamical analysis of "CME watch" data is carried out, for the first time, over a much longer time-range. We use a 12-min-cadence $195 \AA$ data set and observe an "EUV filament" without interruption as it crosses the solar disk.

Solar prominences or filaments, seen at the limb or on the solar disk, which are cool and dense clouds of plasma, have been extensively studied in the $\mathrm{H} \alpha$ (H I) line. The EIT $195 \AA$ bandpass has its greatest sensitivity to coronal plasmas at $1.6 \mathrm{MK}$ and a filament at that temperature appears dark (seen in absorption) on the disk. We define here an "EUV filament" as the coronal void in emission as seen by EIT, that corresponds to a filament channel, not as the $\mathrm{H} \alpha$ filament seen in the EUV (see e.g., Schwartz et al. 2004, for a recent discussion).

Small-amplitude oscillations in prominences or filaments have been detected with periods generally shorter than $90 \mathrm{~min}$ and have been successfully interpreted as magnetohydrodynamical (MHD) waves (see Oliver \& Ballester 2002, for a review). The temperature regime and the spatial and temporal resolution of the "CME watch" EIT data do not seem to be the best suited for detecting such waves in prominences. In the long time sequence that we consider here, we detect however ultra-long-period oscillations in the intensity above an EUV filament. In Sect. 2, the observations and the reduction to data series are described. In Sect. 3, the presence of oscillations in the data is analysed. In Sect. 4, the possible interpretations of the oscillations are discussed.

\section{Data series}

We consider a 12-min-cadence SOHO/EIT $195 \AA$ uninterrupted data set between August 29, 19:36 UT, and September 10, 04:12 UT, 1999. These dates correspond to heliographic longitudes $L$ between $205.76^{\circ}$ and $55.76^{\circ}$ in Carrington Rotation 1953. In this data set, a pair of long parallel quiescent EUV filaments crosses the solar northern hemisphere. For a given EIT $195 \AA$ full-disk solar image, the heliographic projection (i.e. orthographic projection of the solar disk) is deduced, as illustrated in Figs. 1b and c. In addition, the solar Carrington rotation is corrected, so that, in the data series obtained, any feature rotating with the Sun on the solar disk evolves at a nearly stationary position. In Fig. 1c, the overlaid square (S5-N85, $L=60-150$ ) encloses the region where the EUV filaments are observed, and for which a time sequence of projected and derotated images is produced.

In this data set, we observe emission changes at the southern end of the westmost EUV filament, which can be followed for more than 11 days. The image quality of a feature such as an EUV filament increases towards the centre of the disk and away from the limbs, because of the projection effect and the related "limb brightening" in coronal images. The limb 

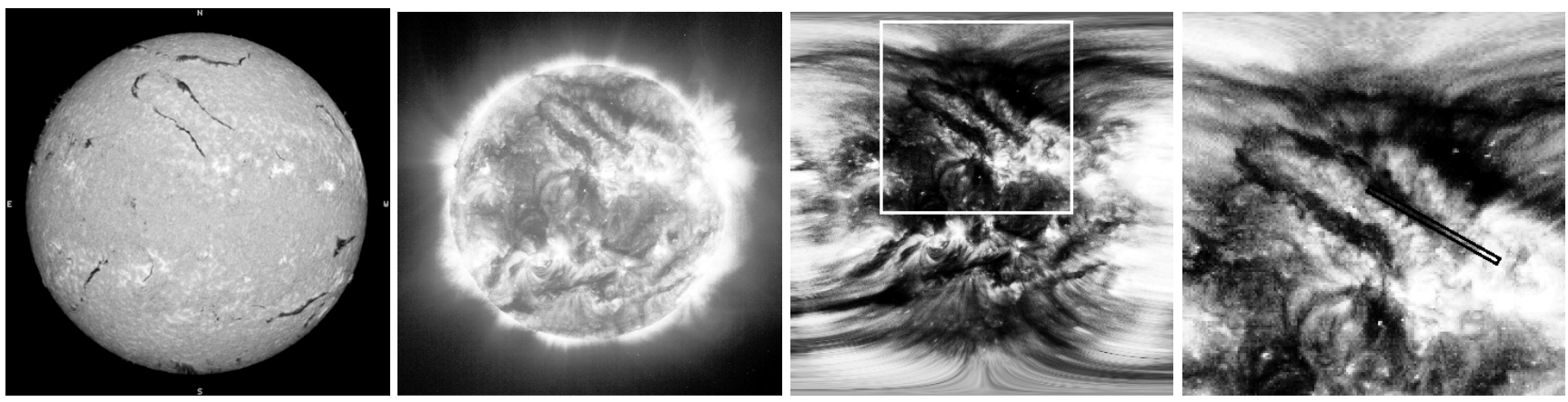

Fig. 1. a) H $\alpha$ solar image taken at 7:04 UT on 6 September 1999 (C)Observatoire de Paris - BASS2000). b) EIT $195 \AA$ full-disk solar image taken at 6:36 UT on the same day. c) The corresponding heliographic projection of the solar disk; the overlaid square (S5-N85, $L=60-150)$ shows a pair of dark long filaments. d) The corresponding snapshot; a rectangular ROI (centred at N37, $L=115$ ) is overlaid in dark.

brightening is caused by the bright loops from active regions and may hide away an EUV filament near the limbs.

A rectangular region of interest (ROI) is chosen along the filament, as shown in the one particular sequence snapshot in Fig. 1d. The ROI is $34.8^{\circ}$ long and $1.4^{\circ}$ wide $\left(1^{\circ} \sim\right.$ $12.15 \mathrm{Mm}$ ). The ROI long axis is directed in the same direction as the filament, i.e. along the neutral line (making an inclination angle of -29.16 degree with respect to the solar equator). A few images with "missing blocks" overlying the ROI are discarded. The final data set includes 1100 images. The sequence is 272.6-h-long and has two main time gaps, one of $2.4 \mathrm{~h}$ and one of $6.5 \mathrm{~h}$. Except for these two gaps, the time cadence is no more than $12 \mathrm{~min}$ for $63.3 \%$ of the data series, and no more than 13 min for $85 \%$.

Nearly $82 \%$ of the EIT image data set have a pixel size corresponding to $2.63 \mathrm{arcsec}$. The rest are half resolution images, present only in the first $47.2 \mathrm{~h}$ of the time sequence. In the high resolution images, the solar disk has a radius $R \sim 370$ EIT pixels, so that projected solar disk maps of length up to $\pi R \sim 1160$ pixels may be obtained. Here, with maps of size $360 \times 360$, a spatial resolution of 2 pixels per degree (corresponding to $6.08 \mathrm{Mm}$ per pixel) is achieved.

\section{Time series analysis}

We first consider the ROI southern quarter (a quarter of the ROI long axis length), where oscillations are observed. The intensity is averaged over all points in the chosen region, thus giving a time series. The series are resampled with the largely prevailing time cadence of $12 \mathrm{~min}(0.2 \mathrm{~h})$, yielding the regular time series $I$ for the averaged intensity in the ROI southern end. Oscillatory variations are visible in $I$, shown as a thin line in Fig. 2a. To examine these oscillations, $I$ is detrended with a 14-h-boxcar running average. The trend, denoted $I_{0}$ and shown as a thick line in Fig. 2a, is mostly due to the limb brightening. The ROI southern end crosses the solar meridian approximately at the time corresponding to the middle of the time series. The asymmetry between the two halves of $I_{0}$ can be explained by variations in the neighbouring solar activity, which is higher in the second half of the time series, and also by the difference between the regions contributing to the limb brightening East (dark filaments) and West (bright active regions) of the ROI southern end. The relative intensity
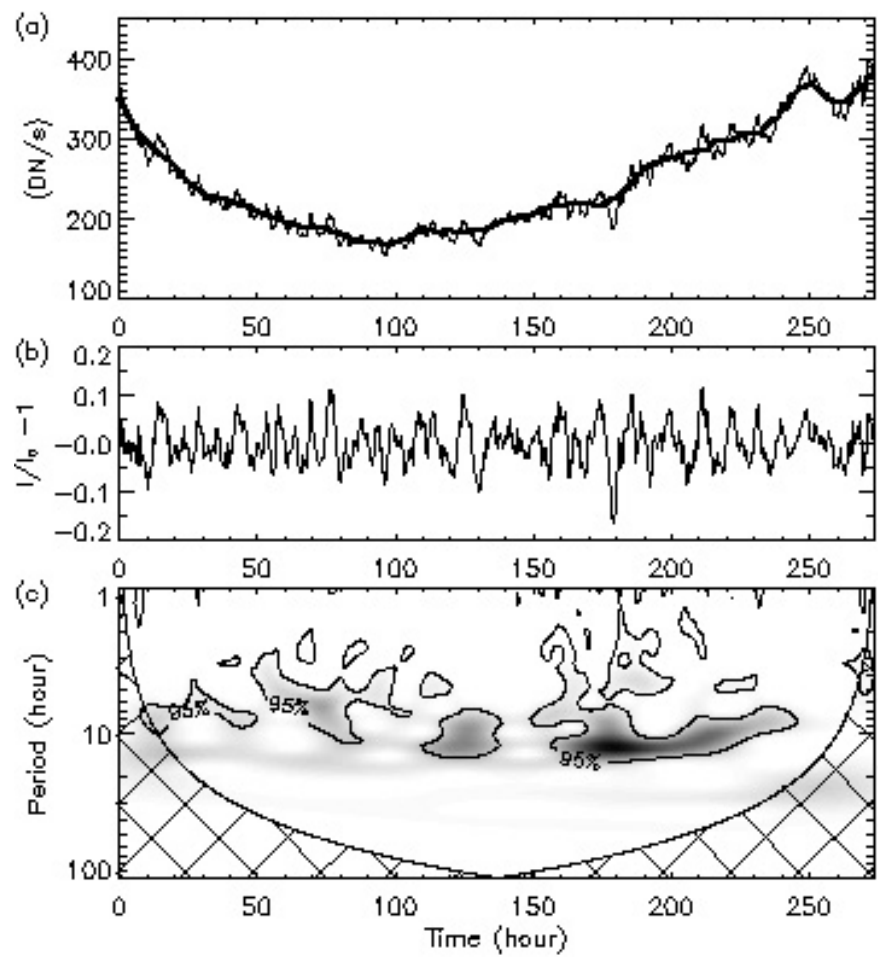

Fig. 2. a) Averaged intensity $I$ in the ROI southern end (thick line) and 14-h-boxcar running average $I_{0}$ (thin line). b) Intensity time series $I / I_{0}-1$. c) Corresponding Morlet wavelet power spectrum: increasing grey level is used to represent increasing power; significant power is within the 95\% confidence level contour (including red noise) and outside the hatched cones of influence.

$I / I_{0}-1$ is shown in Fig. 2b. The amplitude of the intensity variations reaches approximately $10 \%$ of the background intensity. A Morlet wavelet power spectrum of this time series is shown in Fig. 2c, and the corresponding global (time-averaged) wavelet spectrum in Fig. 3.

As illustrated in Torrence \& Compo (1998), the global wavelet spectrum is normalised (with the squared wavelet variance $\sigma^{2}$ ) and compared to the normalised Fourier power spectrum. The global wavelet spectrum gives a smoothed version of the power spectrum. A higher frequency resolution is achieved by computing the Lomb-Scargle periodogram of the non-sampled and detrended time series 


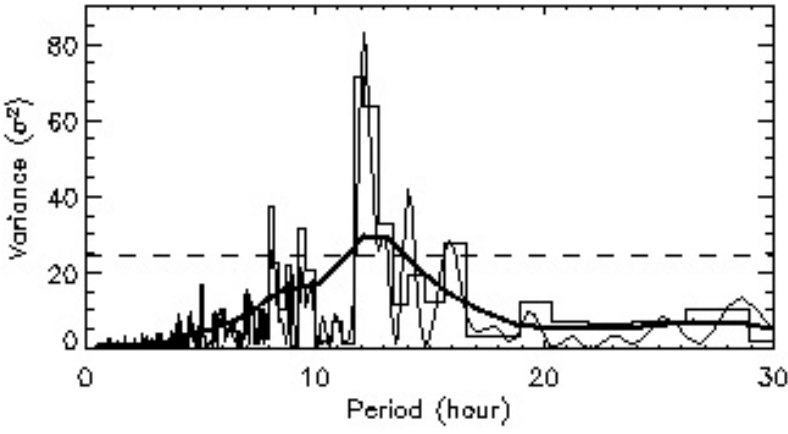

Fig. 3. Lomb-Scargle periodogram (thin solid), normalised Fourier power spectrum (histogram mode) and normalised global wavelet spectrum (thick solid). The horizontal dashed line is the 95\% confidence level for the periodogram.
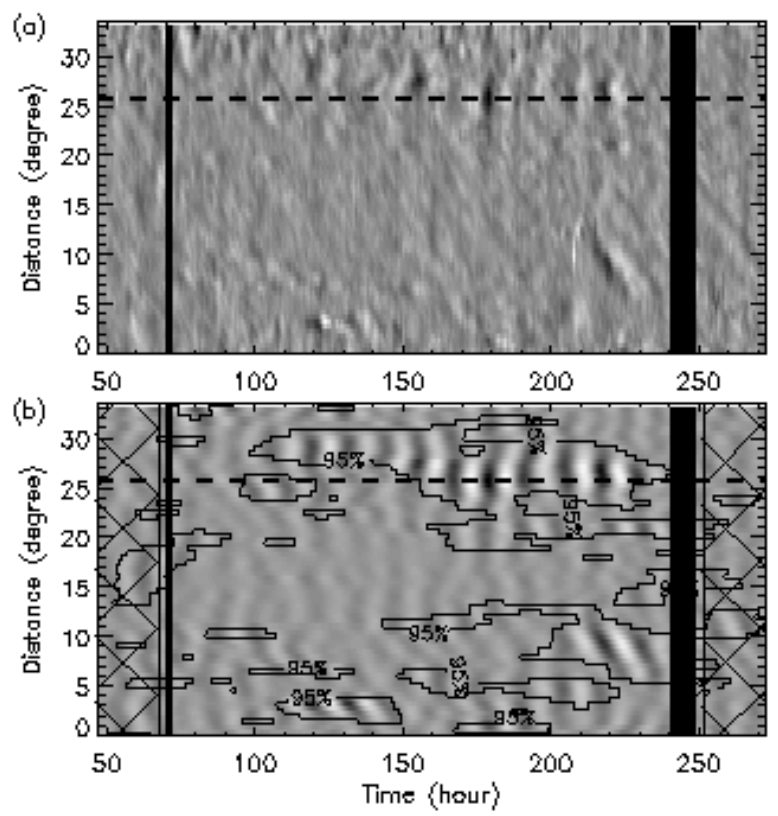

Fig. 4. a) Time-space plot of relative intensity along the ROI long axis. b) Distribution of scale-averaged wavelet transforms over the waveband $12.8 \pm 2 \mathrm{~h}$ along the ROI long axis. Significant power is within the $95 \%$ level contour (including red noise) and outside the hatched cones of influence.

(Scargle 1982; Horne \& Baliunas 1986). The 95\% confidence level for the periodogram was computed according to Bai \& Cliver (1990). The highest peak in the periodogram corresponds to a dominant period of $12.1 \mathrm{~h}$. Lower peaks can be distinguished near 8, 9, 14 and $16 \mathrm{~h}$.

To examine the spatial distribution of the oscillations, we next consider the entire ROI, and run the sequence from Time $=$ $46.8 \mathrm{~h}$. For each pair of adjacent positions along the (69 pixel long) main axis of the ROI, the intensity is averaged and integrated over the shorter dimension. Each (of the 68) time series is then resampled, detrended and analysed with a Morelet wavelet as shown previously. Figure $4 \mathrm{a}$ is a time-space plot of relative intensity, showing the evolution of the detrended intensity above the EUV filament (from East to West). The two main time gaps are represented by dark vertical stripes. The upper delimited section of the plot corresponds to the series
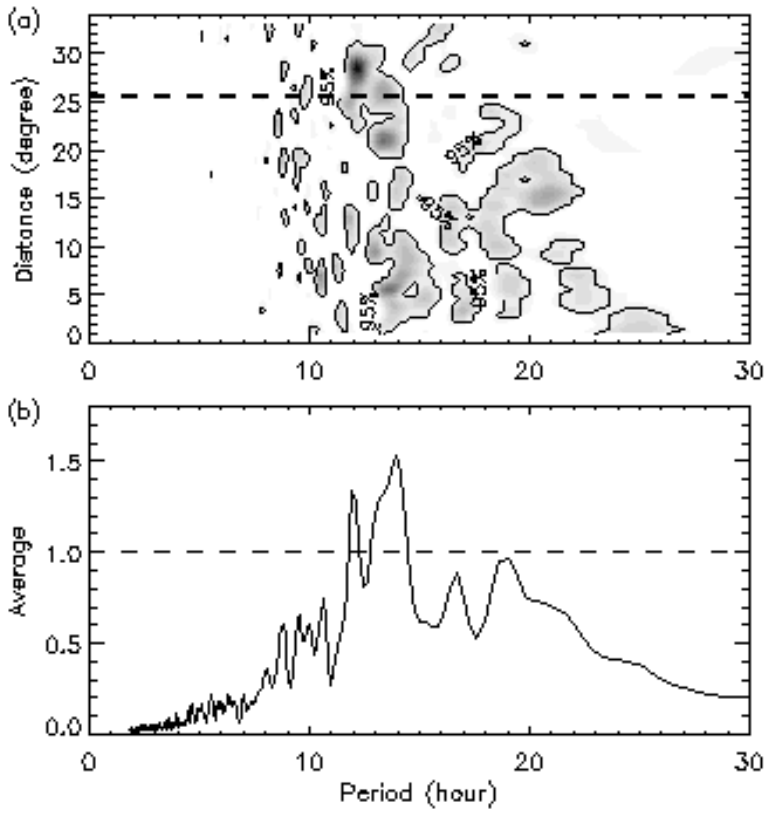

Fig. 5. a) Distribution of the relative Lomb-Scargle periodogram along the ROI long axis. Increasing grey level is used to represent increasing relative power; significant power is within the $95 \%$ level contour. b) Average of the relative spectral powers over distance; the ratio level of 1 is shown as a horizontal dashed line.

at the southern end of the filament; the oscillatory pattern is dominant there, and can also be seen in the adjacent lower regions. In other parts of the filament, no dominant signal may be set apart from the noise, except for an Eastward propagating front, which can be distinguished near the mid-position, starting around Time $=210 \mathrm{~h}$. This front may be associated with a partial eruption involving the pair of EUV filaments, which occurred on September 7, 1999, around 11:12 UT (Time = $207.6 \mathrm{~h}$ ), with the western filament starting to rise in its northern part around 10 UT. Following the partial eruption, the disturbed filaments are subject to much changes in their morphology, with in particular the western filament shrinking to a thin relic.

In order to obtain the spatial distribution of dominant periods above the EUV filament, we perform a Lomb-Scargle periodogram analysis on the non-sampled and detrended time series and take the ratio between the periodogram and the $95 \%$ level for each series. The patchy distribution shown in Fig. 5a is obtained by including more integrated points in each series: the intensity is here a 5-pixel-boxcar running average along the main axis of the ROI, integrated in the transverse direction over twice the original ROI short dimension. Figure 5a shows that oscillations are detected everywhere along the filament with periods ranging from 8 up to $27 \mathrm{~h}$, and confirms that the dominant power is concentrated near the filament's southern end with a dominant period near $12.1 \mathrm{~h}$. Taking the average of the relative spectral powers over distance in Fig. 5b, we see that oscillations with periods near 11.8 and $13.9 \mathrm{~h}$ are statistically dominant throughout the filament.

These periods fit in the dominant waveband of $12.8 \pm 2 \mathrm{~h}$ detected at the ROI southern end. The spatial distribution of the phase for this waveband can be seen in Fig. 4b, which shows 
the distribution of scale-averaged wavelet transforms along the ROI long axis. Oscillatory fronts (alternating bright and dark lanes) are well correlated along the filament, meaning that the oscillations of neighbouring points along the filament are almost in phase. At the ROI southern end, the 12.8-h-period oscillations are standing and are seen continuously for $140 \mathrm{~h}$ (nearly 6 days). Along the filament's body, there are more sporadic and less dominant powers, which correspond to what appear to be aperiodic dynamical events in Fig. 4a, rather more than oscillations.

\section{Discussion}

The discovered periodicities are consistent with several theoretical predictions. To begin with, the ultra-long period oscillations may be interpreted in terms of MHD modes. The prominence model of Joarder \& Roberts (1993) yields global modes of oscillations with the longest possible period given by the slow string mode, viz.

$\tau=\frac{2 \pi \sqrt{l a}}{c_{T 0} \sin \phi}$,

assuming a filament, of width $2 a$ and tying to rigid conducting walls at $\pm l$ ( $l$ corresponds to half the length of magnetic field lines supporting the filament), with $a / l \ll 1 ; c_{T 0}$ is the cusp speed in the filament, $\phi$ is the angle between the magnetic field and the filament long axis. Assuming $c_{T 0} \sim 14.6 \mathrm{~km} \mathrm{~s}^{-1}$ as in Joarder \& Roberts (1993) and having $a<6 \mathrm{Mm}$ from $\mathrm{H} \alpha$ observations (Fig. 1a), we can estimate the length $l$ necessary to explain the observed periods for a range of values $\phi=10-25^{\circ}$. We obtain $l=51-305 \mathrm{Mm}$ for $P=12.1 \mathrm{~h}$. Approximating $2 l \sim \pi r$ to be the length of a semi-circular coronal arcade across the distance $2 r$ between opposite polarities, we obtain $l=79 \mathrm{Mm}$ for $2 r \sim 100 \mathrm{Mm}$. The observed spatial co-existence of oscillations with different periods may be qualitatively explained either as a projection effect or as mode coupling. Consequently, the interpretation of the oscillations as slow string MHD modes is a possibility.

Other possible interpretations are connected with thermal over-stability associated with peculiarities of the cooling/heating function and with the effect of neutrals. Bakhareva et al. (1992) showed that plasma partial ionisation causes dynamic regimes to exist in a prominence, which extends the range of oscillation periods. Numerical studies of prominence condensations (Karpen et al. 2001; Müller et al. 2004) have revealed mass cyclic variations with time periods similar to our observations. As yet, no observational study was carried out to confirm their existence. Such models are one-dimensional, modelling a filament fibril forming on a single loop. The dynamic cycle of condensation formation, drift, and destruction is driven by the process of thermal nonequilibrium. The periods obtained by Karpen et al. (2001) were $22 \mathrm{~h}$ and $15.3 \mathrm{~h}$ for loops of length $340 \mathrm{Mm}$ and $180 \mathrm{Mm}$ respectively. Müller et al. (2004) found periods near $11 \mathrm{~h}$ for a loop of length $100 \mathrm{Mm}$. The possible interpretation of our observations suggests that the spatial and time evolution of emission above and surrounding the EUV filament reflects the evolution of the corona and condensation masses. The observed intensity in the coronal bandpass is a line of sight integration of the absorbing filament and the emitting coronal material above it. To be able to compare the predicted variations in density with our observed intensity amplitude $(<10 \%)$, the line of sight integration should be taken into account (e.g., Schwartz et al. 2004). Finally, the periodic condensations might also trigger the resonance with modes of the filament.

The detailed understanding of the plasma dynamics within, and around, prominences (or filaments) is a crucial step in developing models for heating mechanisms and stability of prominences. Apart from the partial eruption on September 7, 1999, the filaments looked stable as they crossed the solar disk. They erupted again as prominences over the limb on September 14, 1999 (between 5 to 8 UT). The observed cyclic behaviour in the western filament might hold a clue for explaining the eruptions.

In conclusion, cyclic intensity variations, in the band 8-27 h with a dominant period at $12.1 \mathrm{~h}$, are observed for the first time in an EUV filament, and are consistent with several existing theories. The spatial and temporal resolution of EIT, particularly in the high-cadence $195 \AA$ images, offers the best suited capabilities to detect such slow changes, but had never been used for this purpose before. The oscillations are found to be standing along the filament and, consequently, across the magnetic field. There are some non-propagating in phase movements along the filament, suggesting some coupling. Thus, the phenomenon discussed has a global nature and its appropriate modelling should be at least two-dimensional. The theoretical models mentioned above are essentially onedimensional along the field, and are not sufficient to explain the spatial structure of the oscillations.

Acknowledgements. EIT data are courtesy of SoHO/EIT consortium. SoHO is a project of international cooperation between ESA and NASA. Wavelet software was provided by C. Torrence and G. Compo, and is available at URL: http://paos. colorado.edu/research/wavelets/

\section{References}

Bai, T., \& Cliver, E. W. 1990, ApJ, 363, 299

Bakhareva, N. M., Zaitsev, V. V., \& Khodachenko, M. L. 1992, Sol. Phys., 139, 299

Delaboudinière, J.-P., Artzner, G. E., Brunaud, J., et al. 1995, Sol. Phys., 162, 291

Horne, J. H., \& Baliunas, S. L. 1986, ApJ, 302, 757

Joarder, P. S., \& Roberts, B. 1993, A\&A, 277, 225

Karpen, J. T., Antiochos, S. K., Hohensee, M., Klimchuk, J. A., \& MacNeice, P. J. 2001, ApJ, 553, L85

Müller, D. A. N., Peter, H., \& Hansteen, V. H. 2004, A\&A, 424, 289

Oliver, R., \& Ballester, J. L. 2002, Sol. Phys., 206, 45

Scargle, J. D. 1982, ApJ, 263, 835

Schwartz, P., Heinzel, P., Anzer, U., \& Schmieder, B. 2004, A\&A, 421, 323

Thompson, B. J., Gurman, J. B., Neupert, W. M., et al. 1999, ApJ, 517, L151

Torrence, C., \& Compo, G. P. 1998, Bull. Am. Met. Soc., 79, 61 